\title{
PENILE SHADOW ARTEFACT OVERLAPPING FRACTURES
}

\author{
Author: Janet M. Chan, Radiographer Wealth Hospital, Australia \\ Gordon Street, Footscray, Victoria, Australia \\ E-mail: Janet.ml.chan@gmail.com
}

PEER REVIEWED ARTICLE, VOL. 1, NR. 1, p. 26-29 PUBLISHED 27.11.2014

\begin{abstract}
Soft tissue shadows are commonly seen on pelvic radiographs, and radiographers may overlook or are unaware that these shadows could be artefacts. In a case study, shadow of a penis superimposed with the fracture lines at pubic ramus and it was questioned whether a fracture of ramus ischio-pubis is present. Further radiographic views were performed to demonstrate the fractures without any artefact. There are other possible pelvic artefacts that may be seen and neglected on pelvic radiographs, thus it may lead to misdiagnosis of pelvic fracture. This essay should be served as a reminder for radiographers to recognize artefacts and differentiate it from pathology.
\end{abstract}

Keywords: Anatomical over-projection, Anatomical noise, Artefact, Artifact, Pelvic radiography, Penis. Penile shadow. Superimposing organ. Foreign body

\section{Introduction}

An AP pelvis view is routinely requested for a patient suffered from blunt pelvic trauma. It is a common practice to remove all patient's clothing in the area of interest, if possible. Other anatomical parts, such as fat skin and penis, are not often readjusted to avoid overlapping with the pelvis. In this case report, such anatomical artefact is highlighted to remind radiographers that we should remember the negative effect of an anatomical artefact on image quality.

\section{Case Report}

A 41-year-old male patient was sandwiched between doors. He then presented to the emergency department in an acute metropolitan public hospital, located in western Melbourne, Victoria, Australia. A pelvic radiographic examination was requested to query for any fracture. An AP pelvic view confirmed fractures at the pubic tubercle, superior pubic ramus and left ischio-pubic synchondrosis. (Figure 1) These fractures were superimposed with a shadow casted by patient's penis. In order to provide a clearer view of the fractures, the radiographer produced a craniocaudal view of the pubic ramus (Figure 2). In this view, the penis shadow was projected away from the area of interest, so the fractures were shown entirely. 


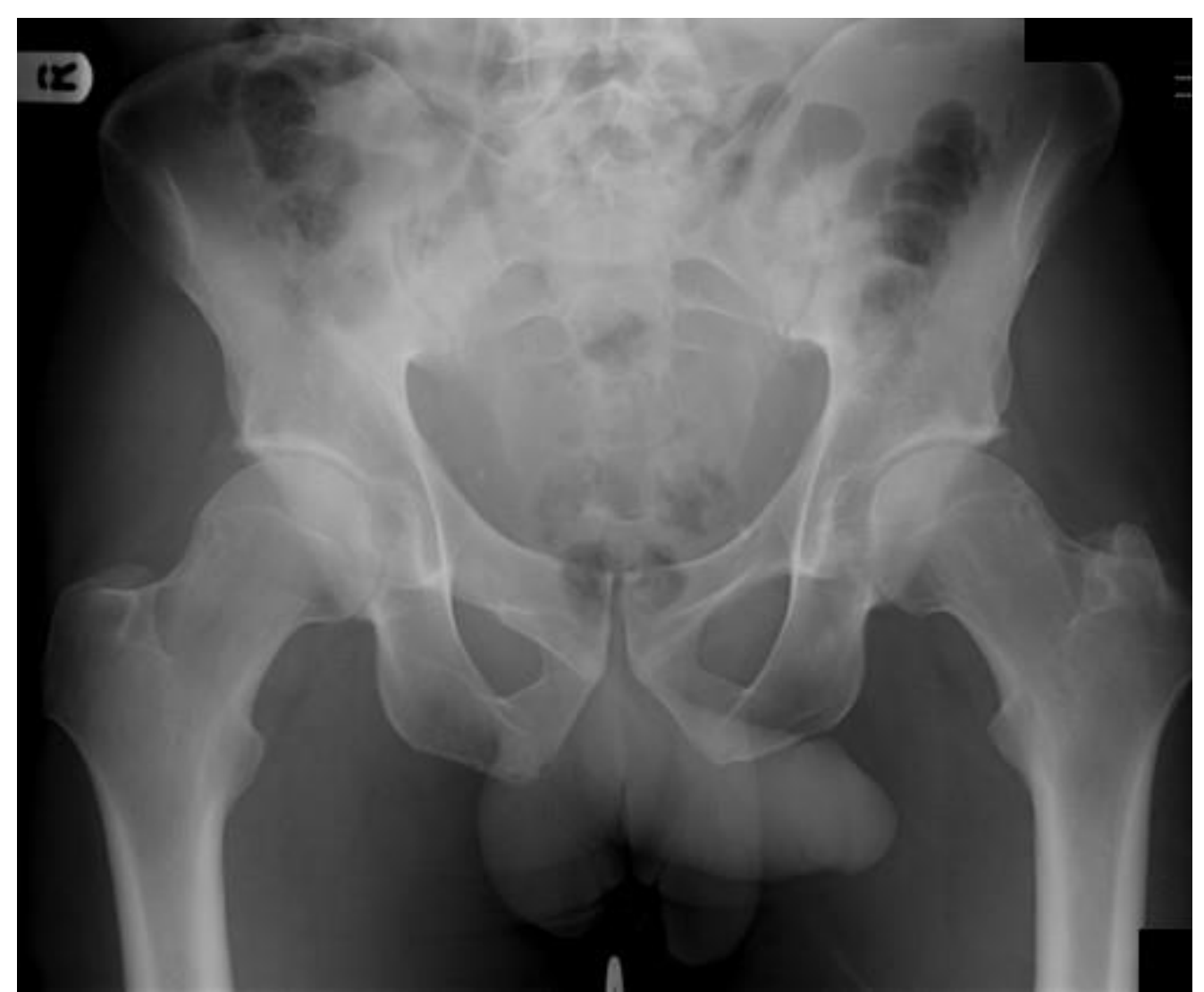

Figure 1: An AP pelvic projection in which the one of several fractures were overlapped with a penis shadow.

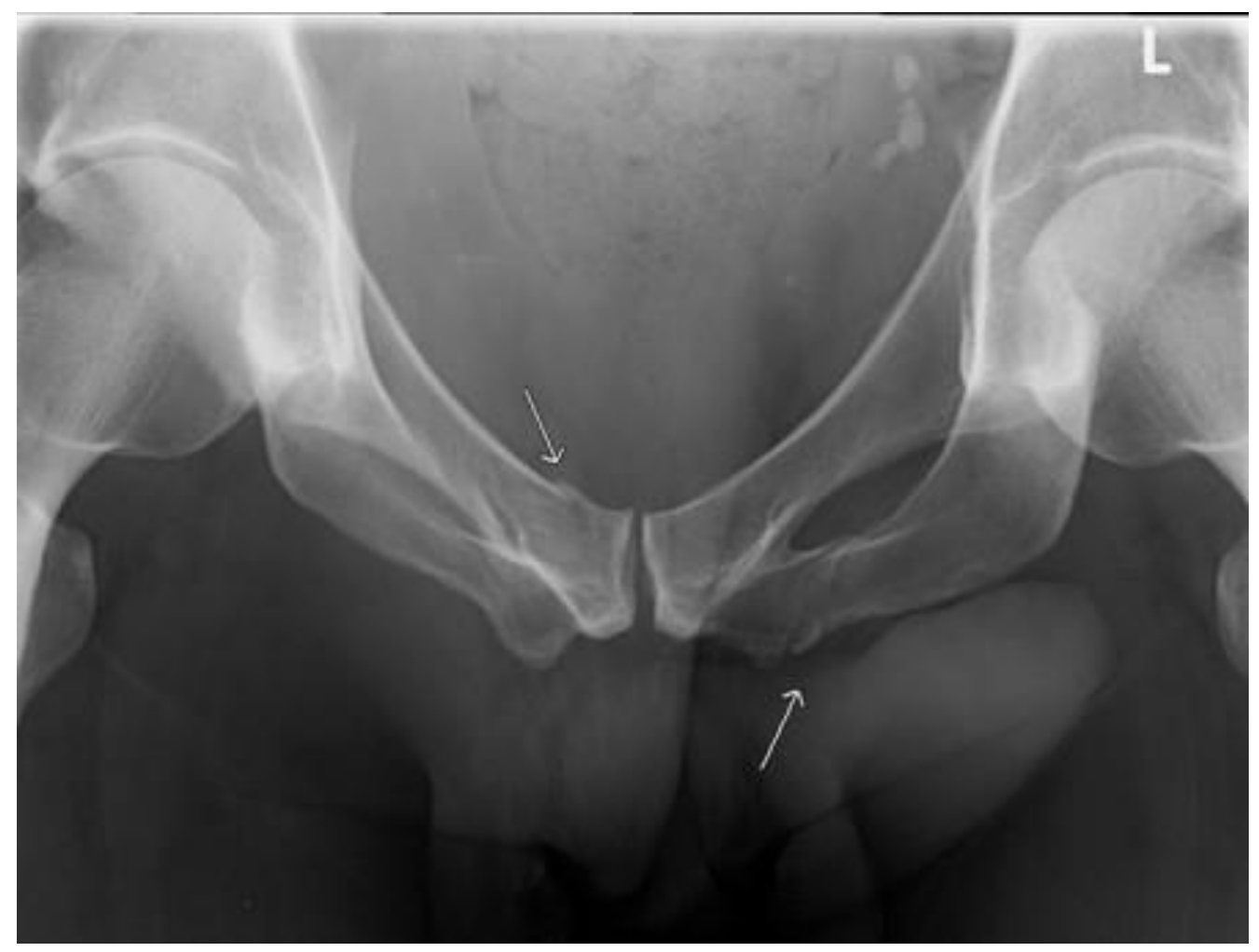

Figure 2: A cranio-caudal view of the pelvic ramus. The penile shadow was projected away from the area of interest. 


\section{Discussion}

"Penile shadow" has been discussed in literatures and was arguably a radiological sign called "John Thomas Sign". It is believed that a penile shadow would point to the side of fracture in a pelvis, and this is considered a positive John Thomas Sign (Murphy, Murphy, \& Heffernan, 2014; Thomas, Lyons, \& Walker, 1998). However, it is inconclusive in this case report because there are bilateral fractures in the pelvis, and the penis pointed to the left side. In fact, the penile shadow superimposed with the fracture lines, so it is undetermined whether the John Thomas Sign would be a hindrance or a useful indicator in the diagnosis.

This example of a penile shadow artefact should be used as a reminder for radiographers to be aware of anatomical artefacts. There are other examples, includes tampon and bowel gas artefacts, which may be encountered in common radiography practice.

A case study highlights an abnormal soft tissue finding on a pelvic radiographic examination of a female patient, and the finding was subsequently concluded to be a tampon (Turton \& Silverman, 1994). Hypothetically, similar objects, such as pads, may potentially degrade the image quality of a pelvic image, in the same way as patients wearing thick clothing or infants wearing wet diapers (Markowitz, 2007; Markowitz, Altes, \& Jaramillo, 2009).

When we approach patients in regard to removing the artefact object, we should maintain professionalism and sensitivity. Cultural and gender concerns should be considered, whether to ask a female patient to remove her tampon or to ask a male patient to adjust his penis position. It is also mindful to remember that in some religions or personal preferences, it may be offensive for a male radiographer to perform an examination on a female patient.

Breathing technique is useful to produce a sharper image of a lateral thoracic spine projection. The same theory could apply to other body parts such as lumbar spine and pelvis. In cases where patients have bowel gas obstructing the view of the area of interest, a long exposure time companied with short mA could be used to blur out the bowel gas, hence producing a clear view of the interested anatomy. Fuller(2011) discussed and endorsed this tehcnique. Although only the lumbar spine examination was suggested by Fuller(2011), the same technique could also be applied in pelvic examination to achieve a similar improved outcome.

\section{Conclusion}

Besides the examples listed above, there are probably other anatomical or foreign body artefacts that could be avoided. It would be an interesting topic to discuss with your colleagues and to see if there are more examples. This case study should be served as a reminder for all radiographers to pay more attention to pelvic images. We should recognize any anatomical artefact, move it if possible, and differentiate it from pathology. Thus, there would be no confusion when radiologists read the images and patients would not be requested for further imaging examinations due to suboptimal image quality.

Radiography Open 2014 Vol. 1

ISSN: 2387-3345 


\section{Conflict of interest}

None declared.

\section{Acknowledgement}

Special thank you to Adam Steward, the tutor radiographer at Western Health, for his mentorship and inspiration.

\section{References}

Fuller, M. J. (2011). Lumbar Spine Breathing Technique. Retrieved from http://www.wikiradiography.net/page/Lumbar+Spine+Breathing+Technique

Markowitz, R. I. (2007). Wet diaper artifact. Pediatric Radiology, 37(12), 1303. http://dx.doi.org/10.1007/s00247-007-0641-8 PMid:17926027

Markowitz, R. I., Altes, T. A., \& Jaramillo, D. (2009). What causes the "wet diaper" artifact? computed tomography and magnetic resonance observations. Clinical Imaging, 33(3), 226-230. http://dx.doi.org/10.1016/j.clinimag.2008.09.013 PMid:19411030

Murphy, I. G., Murphy, C. G., \& Heffernan, E. J. (2014). John Thomas sign--a memorable but misleading sign in hip fractures. Orthopaedics \& Traumatology Surgery \& Research, 100(2), 203206.http://dx.doi.org/10.1016/i.otsr.2013.12.017 PMid:24582209

Thomas, M. C., Lyons, B. D., \& Walker, R. J. (1998). John Thomas sign: common distraction or useful pointer? Medical Journal of Australia, 169(11-12), 670.

PMid:9887926

Turton, D. B., \& Silverman, E. D. (1994). Tampon artifact in bone scintigraphy. Clinical Nuclear Medicine, 19(12), 1103-1104. http://dx.doi.org/10.1097/00003072-199419120-00015

PMid:7874813 